\title{
Is it safe to remove central lines in patients with platelets less than 20,000/uL
}

\author{
Priya Marwah ${ }^{1}$, Stalin Ramprakash ${ }^{2}$, Sai Prasad T R ${ }^{3}$, Mane Gizhlaryan ${ }^{2}$, Deepa Trivedi ${ }^{4}$, \\ Vaibhav Shah ${ }^{4}$, Amit Chitaliya ${ }^{4}$, Sandeep Elizabeth ${ }^{2}$, Rakesh Dhanya ${ }^{3}$, Rajat Agarwal ${ }^{3}$, \\ and Lawrence Faulkner ${ }^{5}$ \\ ${ }^{1}$ Mahatma Gandhi University of Medical Sciences and Technology \\ ${ }^{2}$ Sankalp-People Tree Centre for Pediatric BMT \\ ${ }^{3}$ Sankalp India Foundation \\ ${ }^{4}$ Sankalp-CIMS Centre for Pediatric BMT \\ ${ }^{5}$ Cure2Children Foundation
}

May 7, 2021

\begin{abstract}
Background: Patients with tunnelled CVL may develop blood stream infections which at times are difficult to control without line removal. Concomitant severe thrombocytopenia with platelet transfusion refractoriness is often considered a hard contraindication to any procedure involving a major blood vessel. There is very little literature on the actual clinical risks of tunnelled central line removal in febrile pancytopenic patients. Procedure: We analysed complications and outcomes in all or patients, a total of 52, who underwent CVL removal with platelets $<20,000 / \mathrm{uL}$. Results: No bleeding episodes or unplanned transfusions could be associated with CVL removal. No other complications were also reported. All patients had time to hemostasis within 5 minutes of catheter removal. A total 31 patients were febrile at the time of CVL removal, of which 17 became afebrile within 2 days. We found no difference in response when comparing those whose antibiotic therapy was change/escalation versus those who did not. Removal of CVL under local anaesthesia remained complication-free even at platelets counts less than 20.000/uL. With only RDP support 17 lines were pulled out without any complications when platelets were below 5.000. Conclusion: Our findings suggest that central lines can be safely removed with platelet counts less than $20.000 /$ ul and that this may result in enhanced blood stream infection control. This might be particularly relevant to neutropenic patients in this day and age of MDR germs emergence and paucity of new effective antibiotics.
\end{abstract}

\section{COVER LETTER .}

To,

The Editor-in-Chief,

Pediatric Blood \& Cancer

Dear Sir/Madam,

We are happy to make the first submission submit of our researcharticle with the title "Is it safe to remove central lines in patients with platelets less than 20,000/uL".

We have no conflict of interest to disclose.

We have no suggestions for potential reviewers. We have no preferences for reviewers. 
Central line associated infections may be very difficult to control without removing the lines. Yet, there is very little information published on the safety and complications associated with removing central lines in patients with very low platelets. We have reviewed the complications associated with removal of central lines at very low platelets.

Our findings suggest that it is safe to remove central lines even at very low platelets. We found that lines could be removed safely even with the cover of random donor platelets at very low platelet counts. The place of removing the lines made no difference and removing lines under sedation on bedside was safe. We also found no difference in response between those patients who had antibiotics escalation and those who did not. Our findings suggest that removing central line at relatively low platelets even with limited cover of platelets infusion is safe and effective. We believe these findings are of particular relevance for the readers of PBC who may be faced with the dilemma of central line removal in febrile neutropenic patients. We assessed that our findings are best suited for the readers of PBC and thus the first submission of our findings is being made to PBC.

We hope that PBC finds our work interesting and worth sharing with its readers.

Thank you and kind regards,

Title: Is it safe to remove central lines in patients with platelets less than $20,000 / \mathrm{uL}$ ?

Dr Priya Marwah ${ }^{1}$, Dr Stalin Ramprakash ${ }^{2,3}$, Dr. Sai Prasad T R ${ }^{2,3}$, Dr Mane Gizhlaryan ${ }^{2}$, Dr Deepa Trivedi $^{2,4}$, Dr Vaibhav Shah ${ }^{2,4}$, Dr Amit Chitaliya ${ }^{4}$, Sandeep Elizabeth ${ }^{2,3}$, Rajat Kumar Agarwal ${ }^{2,5}$, Rakesh Dhanya $^{2}$ and Dr Lawrence Faulkner ${ }^{2,6}$

1. Department of Pediatrics, Mahatma Gandhi University of Medical Sciences and Technology, Jaipur

2. Sankalp India Foundation, Bangalore - India

3. Sankalp-People Tree Centre for Paediatric Bone Marrow Transplantation, Bangalore - India

4. Sankalp-CIMS Centre for Paediatric BMT, Ahmedabad - India

5. Jagriti InnoHealth Platforms Pvt. Ltd., Bangalore - India

6. Cure2Children Foundation, Florence - Italy

Corresponding author :

Rajat Kumar Agarwal

\#304-D, Chamundi Emerald, $1^{\text {st }}$ Main, $7^{\text {th }}$ Cross, N S Palya, BTM II, Bangalore -560076

Email: rajat@sankalpindia.net

Contact No: $+91-9880132850$

Authorship Contribution : PM, MG RD, RKA and LF wrote the manuscript. SR, SP, DT, VS, AC and LF participated in medical management. RD, RKA and LF were involved in conception, design, acquisition, analysis, or interpretation of data. All authors critically reviewed and approved the manuscript.

Article type: Research Articles

Word count: Abstract: 234 Main text: 1535.

Tables: 3. Figures: 1. Supporting information: none.

Short running title (50 characters): Removing central lines with platelets less than 20,000/uL?

Keywords:

1. Central Venous Catheter

2. Febrile Neutropenia 
3. Bone Marrow Transplantation

4. Antimicrobial Stewardship

5. Thrombocytopenia

Abbreviation Key:

\begin{tabular}{ll}
\hline CVL & Central venous lines \\
\hline PTH & Sankalp-People Tree Centre for Pediatric BMT, Bangalore \\
CIMS & and Sankalp-CIMS Centre for Pediatric BMT, Ahmedabad \\
CRP & C-reactive protein \\
PCT & Procalcitonin \\
HCT & Hematopoetic stem cell transplantation \\
\hline
\end{tabular}

This article has not been submitted for review/publication elsewhere.

ABSTRACTS (250 words)

Background: Patients with tunnelled CVL may develop blood stream infections which at times are difficult to control without line removal. Concomitant severe thrombocytopenia with platelet transfusion refractoriness is often considered a hard contraindication to any procedure involving a major blood vessel. There is very little literature on the actual clinical risks of tunnelled central line removal in febrile pancytopenic patients.

Procedure : We analysed complications and outcomes in all or patients, a total of 52, who underwent CVL removal with platelets $<20,000 / \mathrm{uL}$.

Results : No bleeding episodes or unplanned transfusions could be associated with CVL removal. No other complications were also reported. All patients had time to hemostasis within 5 minutes of catheter removal. A total 31 patients were febrile at the time of CVL removal, of which 17 became afebrile within 2 days. We found no difference in response when comparing those whose antibiotic therapy was change/escalation versus those who did not. Removal of CVL under local anaesthesia remained complication-free even at platelets counts less than 20.000/uL. With only RDP support 17 lines were pulled out without any complications when platelets were below 5.000 .

Conclusion : Our findings suggest that central lines can be safely removed with platelet counts less than $20.000 / \mathrm{ul}$ and that this may result in enhanced blood stream infection control. This might be particularly relevant to neutropenic patients in this day and age of MDR germs emergence and paucity of new effective antibiotics.

\section{Main text:}

\section{Background}

Indwelling central venous lines (CVL) may develop biofilms and microthrombi, protecting germs from antibiotics ${ }^{1}$. As a result, patients with tunnelled CVL may develop blood stream infections which at times are difficult to control without line removal, particularly if neutropenic. Concomitant severe thrombocytopenia with platelet transfusion refractoriness is often considered a hard contraindication to any procedure involving a major blood vessel. There is however very little literature on the actual clinical risks of tunnelled central line removal in febrile pancytopenic patients not responding to second line broad spectrum parenteral antibiotic therapy, while the safety of USG-guided central line insertion in thrombocytopenic patients is well established $^{2-4}$.

We analysed complications and outcomes in all or patients, a total of 52 , who underwent CVL removal with platelets $<20,000 /$ uL.

\section{Patients and methods}


The medical records available on BMTPlus ${ }^{5}$ of all 294 patients who underwent a total of 302 transplants at the Sankalp-People Tree Centre for Pediatric BMT, Bangalore (PTH) and Sankalp-CIMS Centre for Pediatric BMT, Ahmedabad (CIMS), between August 2015 and March 2020 were analysed to identify patients who had CVL removed while having a baseline platelet count less than 20,000/uL which identified 52 such instances. These 52 transplants had recipients were 31 boys and 21 girls with median age 10.1 (IQR 7.913.0) years. Indication for transplant was severe thalassemia for 46 patients, severe aplastic anemia for 4 patients, Fanconi's anemia for 1 patient and sickle cell disease for 1 patient.

Blood counts, transfusions, maximum daily temperature, clinical notes, culture reports, C-reactive protein $(\mathrm{CRP})$, Procalcitonin (PCT) and antibiotic therapy were extracted for these patients for the duration of 2 day prior to 2 day after CVL removal.

Patients who had a hemoglobin drop of more than $1 \mathrm{gm} / \mathrm{dl}$ on the day after line removal were identified. Defervescence (maximal daily temperature less than $38^{\circ} \mathrm{C}$ ) within 48 hours of line removal was the primary outcome sought. Antibiotics orders were reviewed looking for change on the same day as line removal. Any drop in CRP and PCT values post line removal was also examined for those who had CRP greater than 0.6 $\mathrm{mg} / \mathrm{dl}$ and PCT greater than $0.5 \mathrm{ng} / \mathrm{ml}$ prior to line removal. Culture reports were examined to identify any evidence of central line infection. Clinical notes were reviewed to identify any complication related to central line removal.

CVL was removed at the bedside under local anaesthesia at $\mathrm{PTH}^{5}$ while sedation was employed at CIMS hospital ${ }^{6}$.

Fisher's exact test was performed using $\mathrm{R}$ version 3.5.x to see if there was a difference between those who had a escalation/change in antibiotics versus those who did not at the time of line removal.

\section{Results:}

Of the 52 patients, in 27 the CVL was removed while baseline platelet counts were below 10,000/uL. Only random donor platelets were transfused in 30 patients, single donor platelets alone were infused in 6 patients, both were used in 5 patients and 11 patients had no platelets transfusion on the day of line removal. In all, 9 patients who had a hemoglobin less than $8 \mathrm{gm} / \mathrm{dL}$ received packed red cell transfusions on the day of CVL removal. A hemoglobin drop of more than $1 \mathrm{gm} / \mathrm{dL}$ was observed in 5 patients however none of them had any overt bleeding. No bleeding episodes or unplanned transfusions could be associated with CVL removal. No other complications were also reported. Table I summarises transfusions and significant bleeding. Figure I shows the distribution of central line removal at platelets less than $20,000 / \mu l$ at each of the centre along with the nature of platelet transfusion given.

A total 31 patients were febrile at the time of CVL removal, of which 17 became afebrile within 2 days, 12 continued to have fever and 2 died. Among the 29 patients where the fever response could be evaluated, antibiotics were changed or escalated in 16 of the patients together with line removal. Among these, 10 became afebrile. Of the 13 patients who had no antibiotics escalation, 7 became afebrile. Fisher's exact test performed to compare those whose antibiotic therapy was change/escalation versus those who did not, showed no difference in response $(\mathrm{p}=0.7)$. Positive blood cultures from CVL sampling were found in 16 patients of whom 7 became afebrile within 2 days of line removal. Table II summarises the Fever and infections status.

Only at one of the two centres were enough CRP and PCT reports available for patients who were febrile at the time of CVL removal to be able to determine if there was a response to line removal or not. Of the 18 patients with elevated CRP at CVL removal and follow-up values, 3 showed decrease within 2 days. Of the 5 patients with elevated PCT level and follow-up values 2 showed decrease within 2 days. Table III summarises the CRP and PCT response to line removal.

\section{Discussion}

Hematopoetic stem cell transplantation (HCT) is generally associated with variable periods of severe throm- 
bocytopenia. However, major bleeding (defined as any bleeding other than petechiae or mucosal) occurs in a minority of patients and it is unclear if the administration of platelets is required for central venous catheter removal .

All patients had time to hemostasis within 5 minutes of catheter removal. Our findings were similar to those observed by Stecker et $\mathrm{al}^{7}$. While removing the CVL we did not make an extra effort to remove the polyester cuff if it separated from the catheter as this has been shown to be of no clinical significance in most patients ${ }^{8}$. Moreover, in our subset of patients, primarily with non-malignant diseases, the tunnelled CVL was inserted at the time of conditioning start, so that little or no cuff fibrosis was present at the time of removal. One concern may be the achievement of hemostasis if traction removal fails in a particular patient and a cut down is required to remove the catheter. Unlike the study by Stecker ${ }^{7}$, none of our patients developed this complication. Interestingly, only about five minutes of manual compression were needed to attain complete hemostasis.

Although Stecker et $\mathrm{al}^{7}$ in their study reported adverse events like bruising, minimal blood oozing and discomfort, which are not uncommonly seen, none of our subjects reported any of these events.

Bedside removal of CVL under local anaesthesia remained complication-free even at platelets counts less than $20,000 /$ uL.

In total, 17 lines were pulled out without any complications when platelets were below 5,000 with only RDP transfusions support.

Hemoglobin drop of more than $1 \mathrm{gm} / \mathrm{dL}$ was observed in 5 of the patients but none showed any signs of overt bleeding and did not require any PRBC transfusion post CVL removal.

None of our patients, irrespective of place of removal of CVL, showed any complications.

Of the 31 patients who had fever at the time of CVL removal, 17 (54.8\%) became afebrile within 2 days of removal.

Positive CVL cultures were reported in 16 patients of whom 1 died (6.25\%). A study by Rodriguez et al reported a mortality of $31 \%^{8}$.

A total of 18 patients had elevated CRP levels at the time of CVL removal, of these only 3 showed a decrease in values in the next two days.

Five patients had raised PCT levels at the time of CVL removal, 2 showed decreased PCT levels in the following two days. Even if the mortality associated with CVL infections is still a subject of methodological debates ${ }^{9,10}$, morbidity is well documented and includes severe sepsis and septic shock, septic thrombophlebitis, endocarditis and thromboembolism ${ }^{11}$.

Neutropenia is a major independent risk factor for CRIs, and neutropenic patients with bloodstream infections are at higher risk of mortality compared with non-neutropenic patients ${ }^{12}$. In our cases defervescence and septic markers response seemed to be independent of concomitant neutrophil recovery.

Although patients undergoing allogeneic or autologous HCT are commonly neutropenic, transplantation might further increase the risk of CVL infections independent of the impact of neutropenia. In a recent retrospective study by McDonald and colleagues, on 352 patients undergoing allogeneic HCT, the use of a matched unrelated donor (MUD) and/or haploidentical donor and the use of an ablative conditioning regimen were independently associated with development of CVL infections on multivariate analysis ${ }^{13}$.

The emergence of MDR germs is a growing threat ${ }^{14}$ so any measure limiting the prolonged use of high-end antibiotics is particularly relevant.

In view of increased threat of developing multi drug resistant microorganisms, CVL removal becomes a necessity to reduce morbidity and eventually mortality in patients in ICU settings and patients undergoing HCT. 
Placement of tunnelled central venous catheters has been extensively studied, but we were not able to find any reports on removal-related complications during severe pancytopenia or on the impact of PT, INR, aPTT or platelets transfusions before traction catheter removal.

In conclusion, though our study has limitations in its sample size, it suggests that central lines can be safely removed with platelet counts less than $20,000 /$ ul and that this may result in enhanced blood stream infection control. This might be particularly relevant to neutropenic patients in this day and age of MDR germs emergence and paucity of new effective antibiotics. In our opinion, the risk of infection progression leaving an indwelling CVL in pancytopenic patients with persistent fever not responding to broad spectrum antibiotics far outweigh the minimal risk of severe bleeding associated with CVL removal during severe thrombocytopenia.

Conflict of Interest statement: We declare no conflicts of interest.

Acknowledgements: We would like to thank all our patients and their families. We would also like to thank all the institutions who were involved in caregiving.

\section{References}

1. Gominet M, Compain F, Beloin C, Lebeaux D. Central venous catheters and biofilms: where do we stand in 2017? APMIS . 2017;125(4):365-375. doi:10.1111/apm.12665

2. Cabrini L, Pappacena S, Mattioli L, et al. Administration of blood products to prevent bleeding complications associated with central venous catheter insertion in patients at risk: a systematic review. $B r J$ Anaesth . 2017;118(4):630-634. doi:10.1093/bja/aex060

3. Estcourt LJ, Desborough M, Hopewell S, Doree C, Stanworth SJ. Comparison of different platelet transfusion thresholds prior to insertion of central lines in patients with thrombocytopenia. Cochrane Haematological Malignancies Group, ed. Cochrane Database Syst Rev . Published online December 2, 2015. doi:10.1002/14651858.CD011771.pub2

4. Study collaborative, van de Weerdt EK, Biemond BJ, et al. Prophylactic platelet transfusion prior to central venous catheter placement in patients with thrombocytopenia: study protocol for a randomised controlled trial. Trials . 2018;19(1):127. doi:10.1186/s13063-018-2480-3

5. Agarwal RK, Sedai A, Dhimal S, et al. A prospective international cooperative information technology platform built using open-source tools for improving the access to and safety of bone marrow transplantation in low- and middle-income countries. J Am Med Inform Assoc . 2014;21(6):1125-1128. doi:10.1136/amiajnl2013-002594

6. Sandeep, Priya G, Prasad S, et al. Patterns of central line infections in two bone marrow transplant units in India among children undergoing allogeneic transplant for thalassaemia. Pediatr Hematol Oncol J . 2018;3(3):S56. doi:10.1016/j.phoj.2018.11.161

7. Stecker MS, Johnson MS, Ying J, et al. Time to Hemostasis after Traction Removal of Tunneled Cuffed Central Venous Catheters. J Vasc Interv Radiol . 2007;18(10):1232-1239. doi:10.1016/j.jvir.2007.06.035

8. Rodriguez D, Park BJ, Almirante B, et al. Impact of early central venous catheter removal on outcome in patients with candidaemia. Clin Microbiol Infect Off Publ Eur Soc Clin Microbiol Infect Dis . 2007;13(8):788793. doi:10.1111/j.1469-0691.2007.01758.x

9. O'Grady NP, Alexander M, Burns LA, et al. Guidelines for the Prevention of Intravascular Catheterrelated Infections. Clin Infect Dis . 2011;52(9):e162-e193. doi:10.1093/cid/cir257

10. Gahlot R, Nigam C, Kumar V, Yadav G, Anupurba S. Catheter-related bloodstream infections. Int $J$ Crit Illn Inj Sci . 2014;4(2):162-167. doi:10.4103/2229-5151.134184

11. Soufir L, Timsit J-F, Mahe C, Carlet J, Regnier B, Chevret S. Attributable Morbidity and Mortality of Catheter-Related Septicemia in Critically Ill Patients: a Matched, Risk-Adjusted, Cohort Study.Infect 
Control Hosp Epidemiol . 1999;20(6):396-401. doi:10.1086/501639

12. Lebeaux D, Chauhan A, Létoffé S, et al. pH-mediated potentiation of aminoglycosides kills bacterial persisters and eradicates in vivo biofilms. J Infect Dis . 2014;210(9):1357-1366. doi:10.1093/infdis/jiu286

13. Howell PB, Walters PE, Donowitz GR, Farr BM. Risk factors for infection of adult patients with cancer who have tunnelled central venous catheters. Cancer . 1995;75(6):1367-1375. doi:10.1002/10970142(19950315)75:6<1367::aid-cncr2820750620>3.0.co;2-z

14. Molton JS, Tambyah PA, Ang BSP, Ling ML, Fisher DA. The Global Spread of Healthcare-Associated Multidrug-Resistant Bacteria: A Perspective From Asia. Weinstein RA, ed. Clin Infect Dis . 2013;56(9):13101318. doi:10.1093/cid/cit020

\section{Legends}

List of tables:

1. Table I: Total CVL removals, platelets transfusion and complications vs. platelets range for PTH and CIMS respectively.

2. Table II: Summary of the fever and infections status.

3. Table III: Summary of the CRP and PCT response to line removal.

List of figures:

1. Figure I: Central line removal at platelets less than $20,000 / \mu l$

\section{Hosted file}

Removing CVL lines below 20k plts_Tables and Figures.pdf available at https://authorea. com/users/412464/articles/521172-is-it-safe-to-remove-central-lines-in-patients-withplatelets-less-than-20-000-ul 